\title{
Aloperine protects against cerebral ischemia/reperfusion injury via activating the PI3K/AKT signaling pathway in rats
}

\author{
ZHIMIN LI ${ }^{1 *}$, XING CAO $^{1 *}$, LIGEN XIAO $^{2}$ and RUIJIAO ZHOU ${ }^{1}$ \\ ${ }^{1}$ Department of Neurology, Affiliated Hospital of North Sichuan Medical College; ${ }^{2}$ Department of \\ Cardiothoracic Surgery, Nanchong Central Hospital, Nanchong, Sichuan 637000, P.R. China
}

Received January 12, 2021; Accepted June 23, 2021

DOI: $10.3892 /$ etm.2021.10478

\begin{abstract}
Cerebral ischemia is among the leading causes of death and long-term disability worldwide. The aim of the present study was to investigate the effects of aloperine (ALO) on cerebral ischemia/reperfusion (I/R) injury in rats and elucidate the possible underlying mechanisms. Therefore, a rat model of reversible middle cerebral artery occlusion (MCAO) was established to induce cerebral I/R injury. Following pretreatment with different doses of ALO, the histopathological changes in the brain tissue were evaluated by hematoxylin and eosin staining. The degree of cerebral infarction was determined using by 2,3,5-triphenyltetrazolium chloride staining. Additionally, the levels of oxidative stress- and inflammation-related factors were measured using commercially available kits. Cell apoptosis was assessed by TUNEL staining, while the expression levels of apoptosisand PI3K/AKT signaling pathway-related proteins were determined by western blot analysis. The results demonstrated that ALO alleviated histopathological injury in the brain tissue and the area of cerebral infarction in a dose-dependent manner. Furthermore, significantly reduced levels of reactive oxygen species and malondialdehyde were observed in the ALO-treated rats post-MCAO/reperfusion, accompanied by increased levels of superoxide dismutase, catalase and glutathione. Consistently, treatment with ALO notably decreased the concentration of inflammatory factors, including TNF- $\alpha$, IL-1 $\beta$ and IL-6, in a dose-dependent manner. In addition, ALO attenuated neuronal cell apoptosis, downregulated the expression of Bax and upregulated that of Bcl-2. I/R markedly reduced the expression levels of phosphorylated (p-)PI3K and p-AKT, which were dose-dependently restored
\end{abstract}

Correspondence to: Dr Ruijiao Zhou, Department of Neurology, Affiliated Hospital of North Sichuan Medical College, 234 Fujiang Road, Shunqing, Nanchong, Sichuan 637000, P.R. China E-mail: zhouruijiaozrj@163.com

${ }^{*}$ Contributed equally

Key words: cerebral ischemia/reperfusion, oxidative stress, apoptosis, PI3K by ALO intervention. Collectively, the aforementioned findings indicated that ALO could improve cerebral I/R injury and alleviate oxidative stress, inflammation and cell apoptosis via activating the PI3K/AKT signaling pathway, thus supporting the therapeutic potential of ALO against cerebral I/R injury in ischemic stroke.

\section{Introduction}

Stroke is a heterogeneous disease and among the leading causes of mortality worldwide, while cerebral ischemia is considered as the most common cause of stroke (1). Cerebral ischemia/reperfusion (I/R) injury may irreversibly impair brain function via partly interrupting blood supply to the brain, thus resulting in thrombosis, embolism or hypoperfusion, eventually leading to death or long-term disability (2). To date, there is no effective treatment available for cerebral ischemic stroke. Therefore, the prevention and treatment of cerebral I/R injury is becoming increasingly important in the treatment of ischemic cerebrovascular diseases.

A growing body of literature has suggested that energy metabolism disorders, oxidative stress, inflammation and apoptosis are involved in the pathogenesis of cerebral I/R injury $(3,4)$. Neuroinflammation and oxidative stress are two well-known factors promoting neuronal injury and apoptosis during ischemic stroke (5). Traditional Chinese medicine (TCM) has been widely used to treat cerebral ischemia-related diseases, and has demonstrated notable therapeutic efficacy in clinical practice. TCM comprises several natural active substances (6). Aloperine (ALO) is a novel quinolizidine alkaloid derived from the leaves and seeds of the Sophora alopecuroides L. (Leguminosae) (Fig. 1A) (7). Matrine, one of the major alkaloids of the Sophora plant, has been demonstrated to be effective in ameliorating the histological consequences of middle cerebral artery occlusion (MCAO) in mice through its antioxidant activity and by inhibiting apoptosis (8). As a novel quinolizidine alkaloid derived from Sophora plant, ALO has been recognized as an effective treatment for several neurological diseases. For example, it was previously demonstrated that ALO could attenuate the production of reactive oxygen species (ROS) and cell apoptosis in a cell model of Alzheimer's disease (9). A previous study reported that ALO could protect mice against I/R-induced renal injury via attenuating inflammatory infiltration and 
tubular cell apoptosis (10). Of note, it has also been reported that the protective mechanisms of ALO on cultured rat hippocampal neurons injured by oxygen-glucose deprivation and reperfusion were associated with its antioxidant properties (11). In addition, ALO could ameliorate oxidative damage during early brain injury following subarachnoid hemorrhage via activating the nuclear factor E2-related factor 2/antioxidant response element pathway (12). ALO can easily diffuse across biological membranes and the blood-brain barrier in an energy-deficient environment (11). Therefore, it was hypothesized that ALO may serve a protective role against cerebral I/R injury.

The present study aimed to investigate the protective effects of ALO against cerebral I/R injury using a rat model of reversible $\mathrm{MCAO}$, in the hope that the results may uncover a novel therapeutic option for the prevention of stroke.

\section{Materials and methods}

Animals. A total of 60 specific pathogen-free grade male Sprague-Dawley rats (weight, 200-250 g) were provided by the Shanghai Laboratory Animal Center (Shanghai, China). All rats were housed under standard conditions with a 12-h light/dark cycle, and were given ad libitum access to food and water. The temperature and relative humidity were $22 \pm 2{ }^{\circ} \mathrm{C}$ and $55 \pm 10 \%$, respectively. All animal experimental procedures were approved by the Affiliated Hospital of North Sichuan Medical College (approval no. NSMC201901).

Grouping and drug treatment. Animals were randomly assigned into the following six groups ( $\mathrm{n}=10$ per group): Sham, I/R, I/R + $2 \mathrm{mg} / \mathrm{kg}$ nimodipine (I/R + NIM; positive control group), I/R + $2 \mathrm{mg} / \mathrm{kg}$ ALO, I/R + $25 \mathrm{mg} / \mathrm{kg}$ ALO and $\mathrm{I} / \mathrm{R}+50 \mathrm{mg} / \mathrm{kg}$ ALO. ALO (Santa Cruz Biotechnology, Inc.) was dissolved in saline supplemented with $10 \%$ acetic acid (2.5 $\mathrm{mg} / \mathrm{ml})$, as previously described (10). Rats in the ALO groups were treated with the corresponding doses of ALO by intraperitoneal injection for 7 consecutive days $(4,8,13)$. Animals in the positive control group received intraperitoneal administration of $2 \mathrm{mg} / \mathrm{kg}$ NIM (Shandong Fangming Pharmaceutical Group Co., Ltd.) instead of ALO, while those in the sham group received the same volume of saline (containing $10 \%$ acetic acid). The doses of ALO were determined based on previous studies $(14,15)$. NIM is a dihydropyridine calcium antagonist, which can reduce nerve cell apoptosis through calcium influx, increase the oxygen supply and blood supply to brain tissue by improving the deformability of red blood cells, reducing the permeability of the blood-brain barrier and improving blood rheology, so as to protect cerebrovascular and nerve cells $(16,17)$. NIM can reduce brain inflammation and promote neuronal recovery by inhibiting the expression of inflammatory factors, such as TNF- $\alpha$, IL-1 $\beta$ and IL-6, to relieve the inflammatory response of the body $(18,19)$. NIM has been widely used as the positive control in the cerebral I/R injury (20,21). The MCAO surgery was performed at $2 \mathrm{~h}$ following the last drug administration.

Induction of cerebral I/R injury. Reversible MCAO surgery was performed using an improved Longa-Zea method (22). Following anesthetization with intraperitoneal injection of
$40 \mathrm{mg} / \mathrm{kg}$ pentobarbital sodium, the rats were fixed in a supine position. The left common carotid artery, the external carotid artery and the internal carotid artery were carefully exposed and dissected away from the adjacent nerves. A V-shaped oblique incision was made at the bifurcation of the external and internal carotid arteries with vascular scissors. Then, the artery clamp was reopened, while a paraffin bolt was inserted through the external carotid artery stump into the internal carotid artery until a slight resistance was felt. The time was set as the beginning of the embolism. The upper end of the common carotid artery was then ligated, and the paraffin bolt was gently pulled back through the incision of the external carotid artery $90 \mathrm{~min}$ after the embolism to allow reperfusion. After $24 \mathrm{~h}, \mathrm{I} / \mathrm{R}$ injury was evaluated using H\&E and 2,3,5-triphenyltetrazolium chloride (TTC) staining. Rats in the model group exhibited notable histopathological changes and increased infarct volume, suggesting the success of I/R model. In rats subjected to sham surgery, the arteries were isolated but not ligated or occluded. During the surgical procedure, the body temperature of the rats was maintained at $37 \pm 5^{\circ} \mathrm{C}$ using a heating pad.

$H \& E$ staining. The rats were euthanized with intraperitoneal injection of $200 \mathrm{mg} / \mathrm{kg}$ pentobarbital sodium at $24 \mathrm{~h}$ post-I/R, and their whole brains were removed and washed with saline. Then, the whole brain cortical tissue sample from each animal ( $n=5$ for each group) was soaked in $4 \%$ paraformaldehyde overnight at room temperature. Following dehydration through an ethanol gradient, the tissues were transparentized with xylene, and the brain blocks were embedded in paraffin. The paraffin-embedded tissue samples were then cut into 5- $\mu \mathrm{m}$ coronal sections using a microtome (Leica Microsystems $\mathrm{GmbH})$, deparaffinized in xylene and rehydrated through a descending ethanol series. Following dehydration via a graded ethanol and xylene series, the histological structure of the brain tract was observed under a light microscope (magnification, x400; Olympus Corporation). Finally, three tissue sections from each animal were stained with $H \& E$ using standard techniques according to a previous study (23) and the stained slides were observed under a light microscope (magnification, x400; Olympus Corporation).

Measurement of cerebral infarct volume. To evaluate the cerebral infarct size, TTC staining was performed. Briefly, the rats were euthanatized with $200 \mathrm{mg} / \mathrm{kg}$ pentobarbital sodium at $24 \mathrm{~h}$ post-I/R and thoroughly perfused with saline. The brains ( $\mathrm{n}=5$ for each group) were removed quickly, and the olfactory bulb, cerebellum and brainstem were excised and cut into five 2-mm coronal sections. Subsequently, the brain sections were first immersed in $1 \%$ TTC solution at $37^{\circ} \mathrm{C}$ for $20 \mathrm{~min}$, followed by fixation in $4 \%$ paraformaldehyde for $6 \mathrm{~h}$ at $4^{\circ} \mathrm{C}$. Images of the TTC-stained sections were captured under a light microscope (magnification, x50). TTC stains the non-infarcted region with a deep red pigment, while the infarcted brain area appears white. Infarct size was calculated using ImageJ software (1.52r; National Institutes of Health) and the result was expressed as a percentage of infarct area to total brain area. The infarct volume percentage in the ischemic cerebral hemisphere was calculated using the following equation: [(Contralateral hemisphere volume-volume of 
non-ischemic ipsilateral hemisphere)/contralateral hemisphere volume] x100, as previously reported (24).

Evaluation of oxidative stress-related markers. The brain tissues ( $n=5$ for each group) were obtained at the end of reperfusion and the ischemic hemispheres were then cut into small pieces. These pieces were homogenized with RIPA lysis buffer (Beyotime Institute of Biotechnology), centrifuged at $12,000 \mathrm{x} \mathrm{g}$ for $10 \mathrm{~min}$ at $4^{\circ} \mathrm{C}$, and the supernatant was collected. The levels of ROS (cat. no. E004-1-1), malondialdehyde (MDA; cat. no. A003-1-2) and reduced glutathione (GSH; cat. no. A006-1-1), as well as the activities of superoxide dismutase (SOD; cat. no. cat. no. A001-1-2) and catalase (CAT; cat. no. A007-2-1), were measured in tissue homogenate supernatant with corresponding commercially available kits (Nanjing Jiancheng Bioengineering Institute) using chemical colorimetry, according to the manufacturer's recommendations.

Measurement of inflammatory factors. To determine the levels of inflammation-related factors, including TNF- $\alpha$ (cat. no. F16960), IL-1 $\beta$ (cat. no. F15810) and IL-6 (cat. no. F15870), the brain tissue homogenate supernatant was collected as described above and ELISA was carried out using corresponding kits (Shanghai XiTang Biotechnology Co., Ltd.) according to the manufacturer's instructions. The optical density values were measured at $450 \mathrm{~nm}$ and were read using a plate reader (BioTek Instruments, Inc.).

TUNEL staining. The sections were fixed with $4 \%$ paraformaldehyde for $20 \mathrm{~min}$ at room temperature, deparaffinized, rehydrated and then permeated with proteinase $\mathrm{K}$ for $10 \mathrm{~min}$ at room temperature to increase cell membrane permeability. To evaluate cell apoptosis in the hippocampus post-I/R, TUNEL assay was performed using a commercially available kit (Beyotime Institute of Biotechnology) according to the manufacturer's protocol. The sections were washed by PBS and then incubated with TUNEL solution for $1 \mathrm{~h}$ at $37^{\circ} \mathrm{C}$ in a dark chamber, followed by the counterstaining with 4', 6-diamidino-2-phenylindole (DAPI) for the nuclei for $20 \mathrm{~min}$ at room temperature. A total of three sections from each animal ( $\mathrm{n}=5$ for each group) were analyzed by two investigators, blinded to the origin of the sections. For each section, TUNEL-positive cells were counted in five non-overlapping high-power fields under a light microscope (magnification, $\mathrm{x} 200$ ). The nuclei of healthy cells were stained blue, whereas apoptotic cells with nuclei presented brown/yellow staining were identified as TUNEL-positive cells.

Western blot analysis. For immunoblotting, the brain tissues were lysed on ice and centrifuged at $4^{\circ} \mathrm{C}$ and at $12,000 \mathrm{x} \mathrm{g}$ for $10 \mathrm{~min}$. The supernatant was extracted using RIPA buffer (Beyotime Institute of Biotechnology) containing protease inhibitor cocktail (Beyotime Institute of Biotechnology) and the protein concentration was examined by means of a bicinchoninic acid protein quantification kit (Beyotime Institute of Biotechnology). For each sample, equal amounts of protein (40 $\mu \mathrm{g} / \mathrm{lane})$ were separated by $10 \%$ SDS-PAGE and transferred to a nitrocellulose blotting membrane (Cytiva). Possible non-specific binding was blocked by $5 \%$ skimmed milk for
$1.5 \mathrm{~h}$ at room temperature and the membranes were then incubated overnight at $4^{\circ} \mathrm{C}$ with specific primary antibodies. On the following day, horseradish peroxidase-conjugated secondary antibody (cat. no. 7074S; 1:3,000; Cell Signaling Technology, Inc.) were added to the membranes and incubated for $1 \mathrm{~h}$ at room temperature. Protein bands was scanned and visualized using an enhanced chemiluminescence detection system (Thermo Fisher Scientific, Inc.). The relative intensity of each band was quantified using ImageJ software (1.52r; National Institutes of Health). The protein expression was normalized to GAPDH levels. Anti-Bax (cat. no. 14796S; 1:1,000), anti-phosphorylated (p-)PI3K (cat. no. 17366S; 1:1,000), anti-PI3K (cat. no. 4249S; 1:1,000), anti-p-AKT (cat. no. 4060T; 1:1,000), anti-AKT (cat. no. 4691T; 1:1,000) and anti-GAPDH (cat. no. 5174T; 1:1,000) antibodies were obtained from Cell Signaling Technology, Inc. Anti-Bcl-2 (cat. no. sc-7382; 1:1,000) antibody was provided by Santa Cruz Biotechnology, Inc.

Statistical analysis. All experiments were repeated independently in triplicate. Data are presented as the mean \pm standard deviation. One-way analysis of variance was used to assess multiple differences, followed by a Tukey's post hoc test with GraphPad Prism 6.0 software (GraphPad Software, Inc.). $\mathrm{P}<0.05$ was considered to indicate statistically significant differences.

\section{Results}

ALO treatment significantly alleviates brain injury and reduces the cerebral ischemic area in MCAO rats. To investigate the potential neuroprotective effect of ALO in cerebral ischemic stroke, the histopathological changes in the brain tissues were evaluated using $\mathrm{H} \& \mathrm{E}$ staining following treatment of MACO rats with ALO. In the sham group, the outline of the cortex neurons was clear and their structure was compact, with abundant cytoplasm. In addition, I/R injury was associated with unequivocal signs of pyknotic and shrunken nuclei, nuclear loss and numerous vacuolated spaces, which were markedly restored following ALO preconditioning in a dose-dependent manner (Fig. 1B and C; $\mathrm{P}<0.001$ ). Additionally, treatment with high doses of ALO had the same effects as NIM treatment. Consistently, as shown in Fig. 1D and E, I/R challenge notably increased the infarct volume compared with the sham group $(\mathrm{P}<0.001)$, while significantly decreased infarct volumes were observed in the ALO and NIM intervention groups compared with the $I / R$ group $(\mathrm{P}<0.001)$. These findings suggested that ALO treatment significantly improved brain injury and the cerebral ischemic area in MCAO rats.

ALO preconditioning ameliorates $M C A O /$ reperfusion-induced oxidative stress and inflammation. Subsequently, the levels of oxidative stress- and inflammation-related markers in rat brain tissue homogenates were assessed using commercially available kits. As shown in Fig. 2A-E, I/R injury markedly increased the contents of ROS and MDA $(\mathrm{P}<0.001)$, while it reduced SOD, CAT and GSH compared with the sham group $(\mathrm{P}<0.001)$. Furthermore, compared with the $\mathrm{I} / \mathrm{R}$ group, pretreatment with ALO dose-dependently decreased ROS and MDA and increased SOD, CAT and GSH $(\mathrm{P}<0.01, \mathrm{P}<0.001)$. 
A<smiles>C1=C2CCCNC2[C@H]2C[C@@H]3CCCCN3C[C@@H]1C2</smiles>

Chemical structure of ALO
B

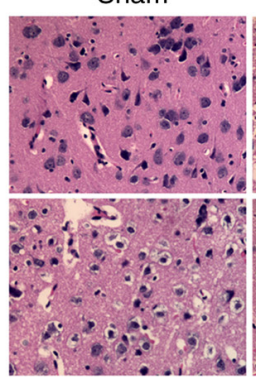

10
$\mathrm{I} / \mathrm{R}$

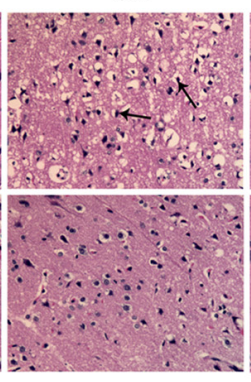

25

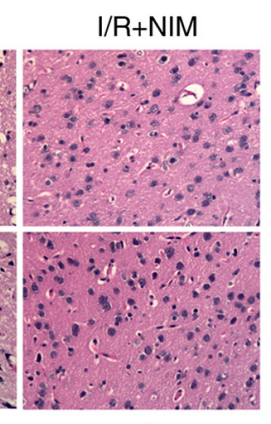

50
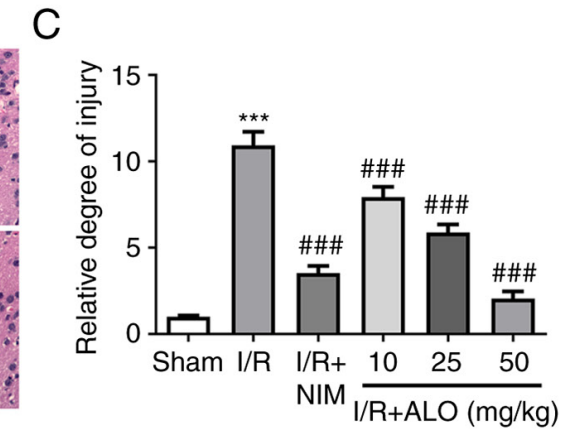

l/R+ALO (mg/kg)

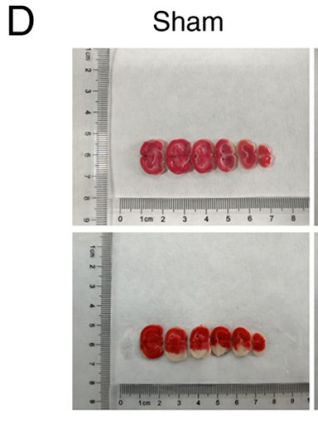

10

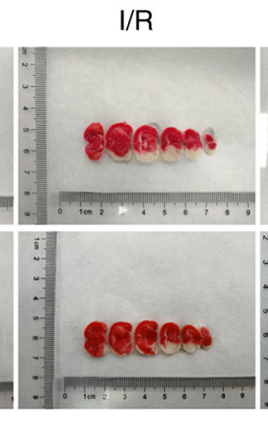

25
$\mathrm{I} / \mathrm{R}+\mathrm{NIM}$

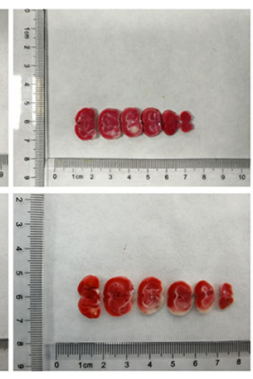

50

$\mathrm{I} / \mathrm{R}+\mathrm{ALO}(\mathrm{mg} / \mathrm{kg})$

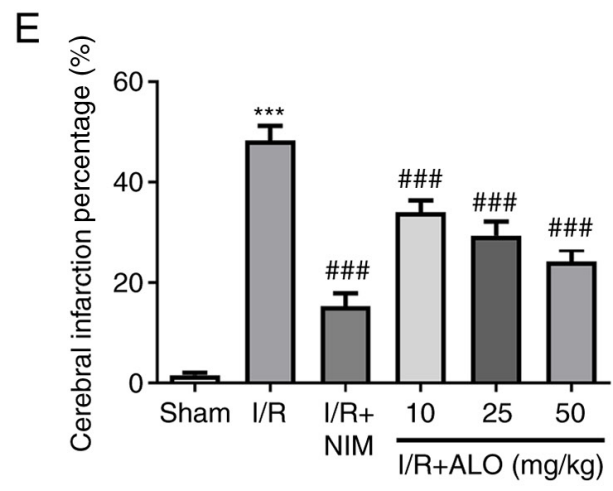

Figure 1. ALO treatment markedly relieves brain injury and reduces the cerebral ischemic area in MCAO rats. (A) Chemical structure of ALO. (B and C) Histopathological changes in the brain tissues were evaluated using hematoxylin-eosin staining following treatment of MACO rats with ALO. Magnification, $x$ 400. (D and E) Cerebral infarction volume in each group was evaluated using 2,3,5-triphenyltetrazolium chloride staining. $\mathrm{n}=5$ per group. ${ }^{* * * *} \mathrm{P}<0.001$ vs. sham group; ${ }^{\# \# /} \mathrm{P}<0.001$ vs. I/R group. ALO, aloperine; MCAO, middle cerebral artery occlusion; I/R, ischemia/reperfusion; NIM, nimodipine.

Simultaneously, the levels of inflammatory factors, including TNF- $\alpha$, IL- $1 \beta$ and IL-6, were notably enhanced following $\mathrm{I} / \mathrm{R}$ exposure compared with the sham group $(\mathrm{P}<0.001)$, whereas ALO or NIM preconditioning markedly reduced the levels of the aforementioned inflammatory factors $(\mathrm{P}<0.001)$. These findings indicated that ALO treatment markedly attenuated oxidative stress and neuroinflammation in rats post-MCAO/reperfusion.

ALO intervention inhibits cell apoptosis in the brain tissues of rats following MCAO/reperfusion injury. To evaluate the effect of ALO on cell apoptosis post-MCAO/reperfusion, TUNEL staining was performed on rat brain sections. A significant increase in the number of apoptotic cells was observed in the I/R group compared with the sham group (Fig. 3A and B; P 0.001). Of note, treatment with ALO dose-dependently reduced the number of apoptotic cells in the brain tissues of rats with $\mathrm{MCAO} /$ reperfusion injury $(\mathrm{P}<0.001)$. Concurrently, compared with the sham group, the expression levels of the pro-apoptotic protein Bax was notably upregulated in the I/R group, accompanied by decreased expression of the anti-apoptotic protein $\mathrm{Bcl}-2$ (Fig. 3C; P<0.001). Importantly, the effect of MCAO/reperfusion on the expression of apoptosis-related proteins was restored by ALO preconditioning in a dose-dependent manner $(\mathrm{P}<0.001)$. These findings indicated that ALO could attenuate neuronal apoptosis in response to cerebral I/R injury in rats.
$A L O$ protects against cerebral $I / R$ injury via activating the PI3K/AKT signaling pathway. To uncover the potential mechanisms underlying the role of ALO in cerebral ischemic stroke, the expression levels of PI3K/AKT pathway-related proteins were determined by western blot analysis. As shown Fig. 4, I/R injury significantly reduced the protein expression levels of p-PI3K and p-AKT compared with the sham group $(\mathrm{P}<0.001)$, whereas this inhibitory effect was reversed following treatment with ALO or NIM $(\mathrm{P}<0.05$ and $\mathrm{P}<0.001$, respectively). Overall, the aforementioned data suggested that ALO treatment could protect against cerebral I/R injury via activating the PI3K/AKT signaling pathway.

\section{Discussion}

Brain tissue is highly sensitive to injury and neuronal apoptosis due to I/R (25). TCM has become increasingly important in the treatment of cerebral I/R injury. It has been reported that an alkaloid-free ethyl acetate extract from the root of Sophora flavescens Ait. exerted neuroprotective effects on focal cerebral ischemia in rats (26). In addition, oxymatrine and matrine, as the main alkaloids extracted from the traditional Chinese herb, Sophora flavescens Ait., have been shown to be effective in improving hypoxic-ischemic brain injury in neonatal rats and MCAO mice by antioxidant and anti-apoptotic activities $(8,27)$. As a novel quinolizidine alkaloid derived from Sophora alopecuroides L., ALO has important beneficial effects in the treatment of neurological diseases. ALO was 

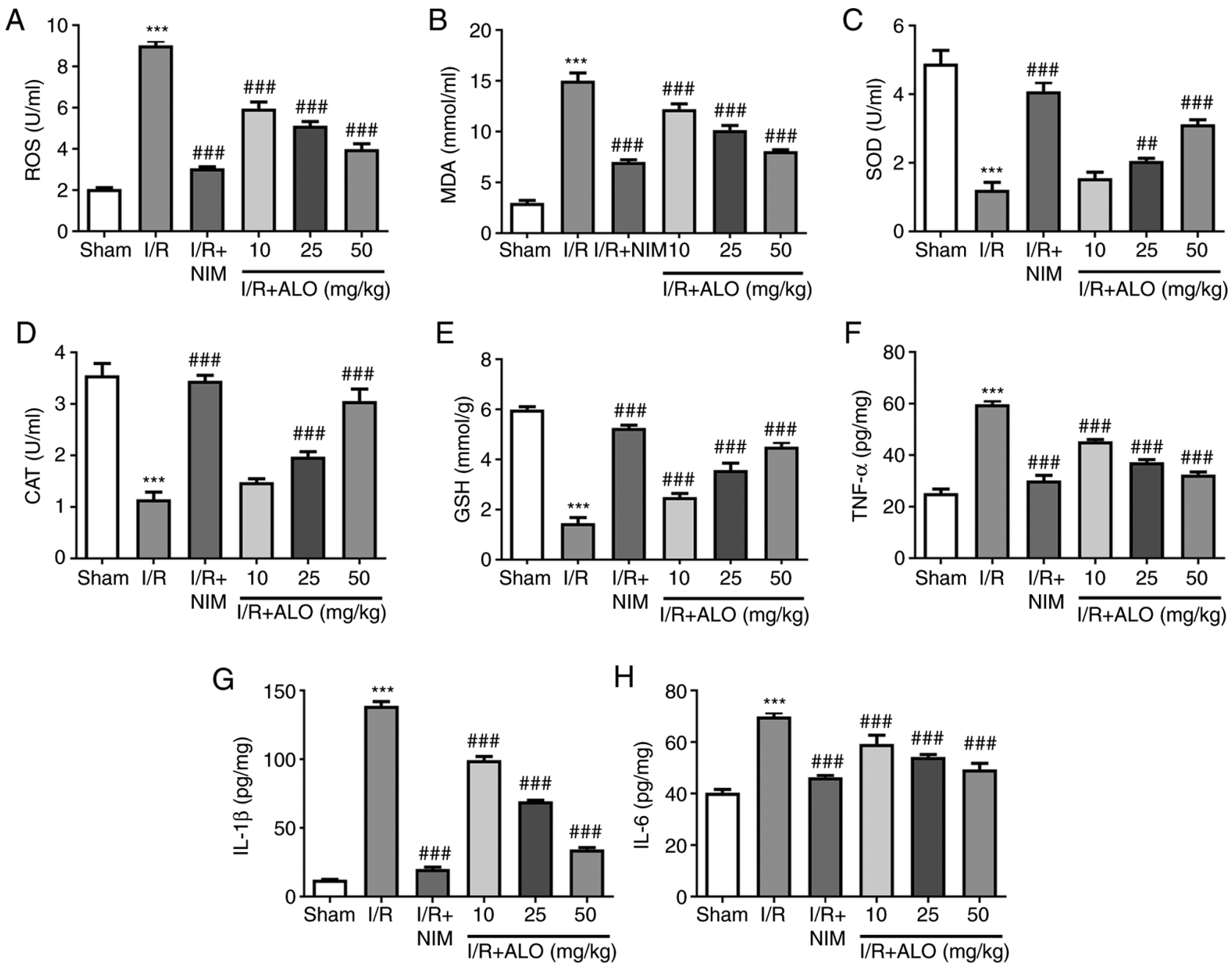

$\mathrm{H}$

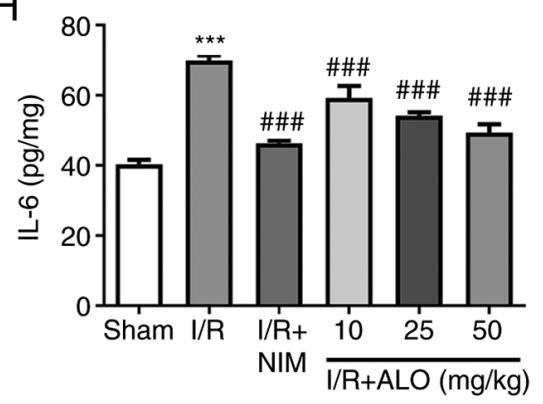

Figure 2. ALO preconditioning alleviates the MCAO/reperfusion-induced oxidative stress and inflammation. The levels of (A) ROS, (B) MDA, (C) SOD, (D) CAT and (E) GSH were measured using commercially available kits. The levels of (F) TNF- $\alpha$, (G) IL-1 $\beta$ and (H) IL-6 were assessed by ELISA. $\mathrm{n}=5$ per group. ${ }^{* * *} \mathrm{P}<0.001$ vs. sham group; ${ }^{\# \#} \mathrm{P}<0.01$ and ${ }^{\# \# \|} \mathrm{P}<0.001$ vs. I/R group. ALO, aloperine; ROS, reactive oxygen species; MDA, malondialdehyde; SOD, superoxide dismutase; CAT, catalase; GSH, glutathione; I/R, ischemia/reperfusion; NIM, nimodipine.

shown to alleviate oxygen-glucose deprivation and reperfusion-induced cultured rat hippocampal neuron injury and ameliorate early brain injury following subarachnoid hemorrhage $(11,12)$. To the best of our knowledge, the present study was the first to investigate the effects of ALO on cerebral I/R injury in rats subjected to MCAO and its potential regulatory mechanisms. The results of the present study demonstrated the beneficial effect of ALO against neuronal injury in a rat model of MCAO/reperfusion, and further suggested that these protective effects may be mediated by the PI3K/AKT pathway.

The pathophysiological mechanisms underlying neuronal injury in cerebral I/R are complex and multifactorial, and a considerable body of evidence has demonstrated that oxidative stress plays a dominant role in the occurrence and development of this condition $(28,29)$. It has been reported that increased production of ROS and lipid peroxidation can be detected at the early stages of cerebral I/R $(13,30)$. MDA, the end product of lipid peroxidation, is a marker of oxidative stress. SOD and CAT are crucial enzymes of the antioxidant defense system produced under oxidative stress, which together protect neuronal cells against ROS-induced cell death $(31,32)$. GSH, a tripeptide with a free sulfhydryl group, reduces oxidative stress and helps to maintain the normal reduced redox state in cells via directly interacting with ROS (33). Emerging evidence has suggested that the excessive accumulation of ROS accompanied by an exhausted antioxidant defense system are involved in the pathogenesis of cerebral I/R injury (34). ALO is a quinolizidine alkaloid derived from the leaves and seeds of the Sophora plant, which has several pharmacological functions in human diseases through its antioxidant activity $(7,35)$. Compelling evidence has indicated that ALO exerts protective effects against hydrogen peroxide-induced oxidative stress and apoptosis in human retinal pigment epithelial cells (36). In addition, a study revealed that ALO could inhibit ROS formation and cell apoptosis in an Alzheimer's disease cellular model (9). Additionally, the significant neuroprotective effects of ALO on oxygen-glucose deprivation/reperfusion-injured neonatal rat primary cultured hippocampal neurons were attributed to its antioxidant activity (11). In the present study, pretreatment of rats post-MCAO/reperfusion with ALO markedly reduced the levels of oxidative stress, supporting the potential protective effects of ALO against cerebral I/R injury.

Accumulating evidence has shown that the inflammatory response is triggered immediately after ischemia, thus resulting 
A

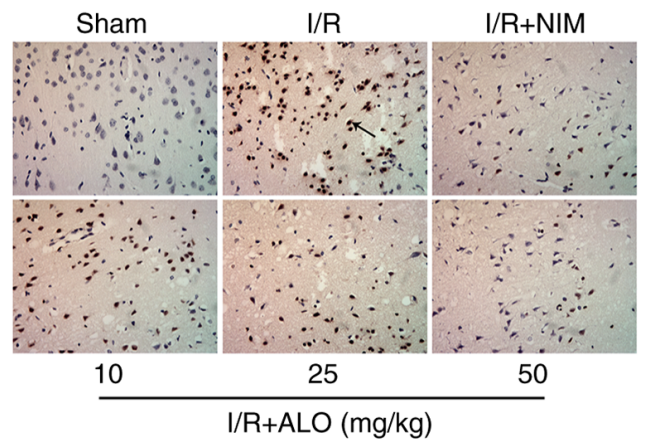

B

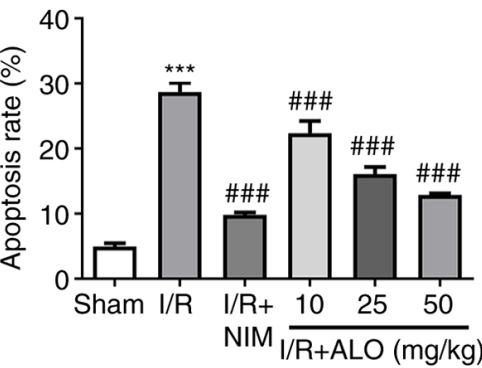

C
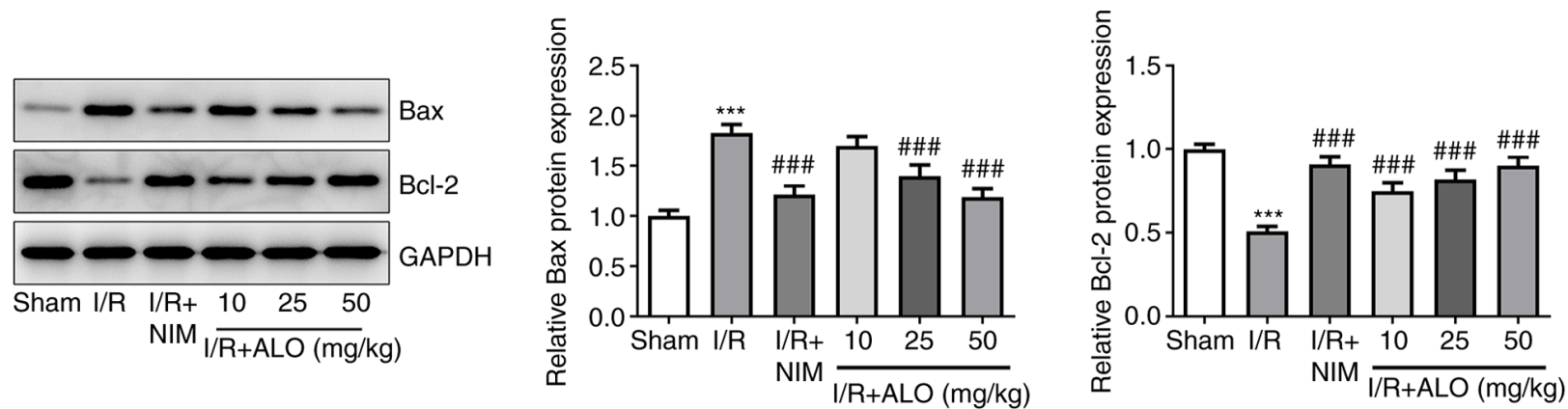

Figure 3. ALO intervention attenuates cell apoptosis in brain tissues of rats following MCAO/reperfusion injury. (A and B) Cell apoptosis in the ischemic cortex was evaluated by TUNEL staining. Magnification, x200. (C) Apoptosis-associated protein expression was determined by western blot analysis. $\mathrm{n}=5$ in each group. ${ }^{* * *} \mathrm{P}<0.001$ vs. sham group; ${ }^{\# \#} \mathrm{P}<0.001$ vs. I/R group. ALO, aloperine; MCAO, middle cerebral artery occlusion; I/R, ischemia/reperfusion; NIM, nimodipine.
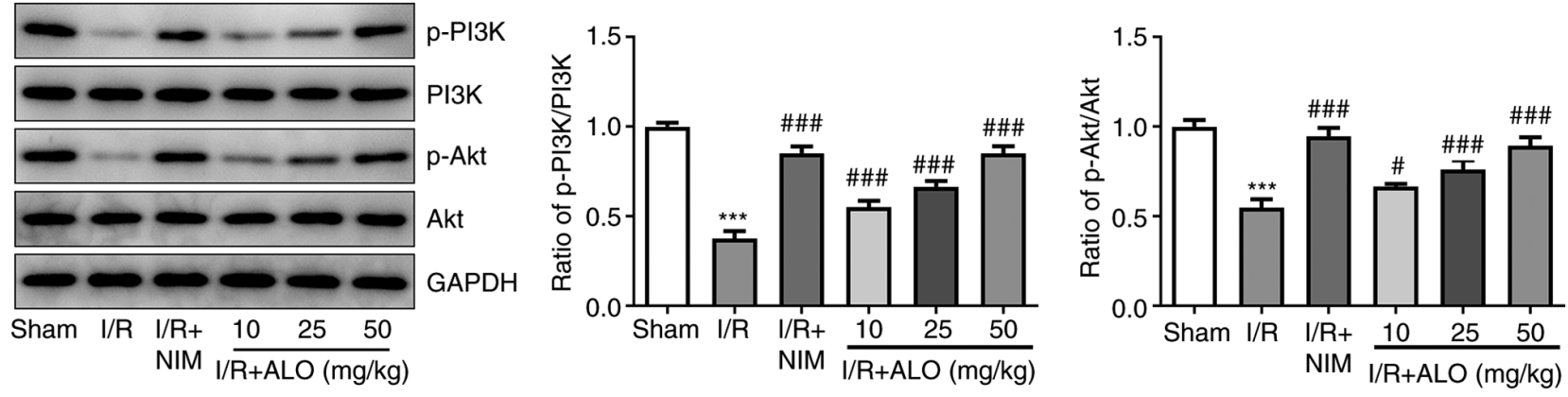

Figure 4. ALO protects against cerebral I/R injury via activating the PI3K/AKT signaling pathway. The expression levels of the PI3K/AKT signaling-related proteins were detected by western blot analysis. $\mathrm{n}=5$ in each group. ${ }^{* * *} \mathrm{P}<0.001$ vs. sham group; ${ }^{\#} \mathrm{P}<0.05$ and ${ }^{\# \# \#} \mathrm{P}<0.001$ vs. $\mathrm{I} / \mathrm{R}$ group. ALO, aloperine; $\mathrm{I} / \mathrm{R}$, ischemia/reperfusion; NIM, nimodipine; p-, phosphorylated.

in the deterioration of delayed cerebral injury and poor functional recovery of neurons (37). Oxidative stress increases the levels of inflammatory cytokines, including TNF- $\alpha$, IL-6 and IL-1 $\beta$, and is closely associated with the injury caused by the excessive secretion of these mediators (38). A cascade of physiological events during the inflammatory responses after cerebral I/R may result in neuronal cell death and neurological dysfunction, eventually leading to brain injury $(39,40)$. Apoptosis is considered as an important type of neuronal cell death that occurs after cerebral I/R injury $(41,42)$. A study revealed that ALO could attenuate human pulmonary vascular smooth muscle cell proliferation via suppressing inflammatory response (43). Mao et al (44) demonstrated that ALO could restrain cardiomyocyte apoptosis and alleviate coronary microembolization-induced rat myocardial injury. A previous study demonstrated that ALO could protect mice against I/R-induced renal injury via suppressing inflammatory infiltration and tubular cell apoptosis (10). Consistent with the aforementioned findings, the present study revealed that cerebral MCAO/reperfusion could markedly enhance the secretion of inflammatory cytokines (TNF- $\alpha$, IL-6 and IL-1 $\beta$ ) and promote cell apoptosis in brain tissues. These effects were effectively inhibited following treatment with ALO, indicating that the protective effect of ALO on cerebral I/R injury could be associated with the inhibition of inflammation and apoptosis.

The PI3K/AKT signaling pathway is a known regulator of a wide range of cellular functions, such as proliferation, oxidative stress, inflammation and apoptosis (45). Mounting evidence indicates that the activation of PI3K/AKT signaling plays an important role in cerebral I/R injury via promoting the repair and survival of ischemic nerve cells $(46,47)$. In the 
nervous system, PI3K is involved in the survival and differentiation of neuronal cells, and PI3K activation can modulate the expression of downstream target genes via activating AKT expression (48). A previous study demonstrated that AKT could exert a critical protective effect on cerebral I/R injury in rats, since its overexpression was found to reduce the volume of the infarcted brain tissue (49). Therefore, the development of effective PI3K/AKT activators may be a focus of future research on ischemic cerebrovascular diseases. ALO was found to attenuate coronary microembolization-induced myocardial injury in rats via activating the PI3K/AKT signaling pathway (44). Additionally, ALO was shown to regulate inflammatory responses in colitis via suppressing the PI3K/AKT pathway in a PP2A-dependent manner (50). The present study revealed that ALO treatment markedly upregulated the expression of $\mathrm{p}-\mathrm{PI} 3 \mathrm{~K}$ and $\mathrm{p}-\mathrm{Akt}$ in rats post-MCAO/reperfusion, suggesting that ALO may protect against cerebral I/R injury via activating the PI3K/AKT signaling pathway.

Taken together, the findings of the present study demonstrated that ALO exerted anti-neuroinflammatory, antioxidant and anti-apoptotic effects during cerebral I/R injury via activating the PI3K/AKT signaling pathway. These data support the therapeutic potential of ALO in cerebral ischemic stroke. However, the lack of behavioral measures and neurological deficit scores constitute limitations of the present study, and will be addressed in future studies.

\section{Acknowledgements}

Not applicable.

\section{Funding}

No funding was received.

\section{Availability of data and materials}

The datasets used and/or analyzed during the current study are available from the corresponding author on reasonable request.

\section{Authors' contributions}

ZL, XC and LX searched the literature, designed the experiments and conducted the experiments. XC and RZ analyzed and interpreted the data. ZL wrote the manuscript. RZ revised the manuscript. ZL and XC confirmed the authenticity of all the raw data. All the authors have read and approved the final version of the manuscript.

\section{Ethics approval and consent to participate}

All animal experimental procedures were approved by the Animal Care and Use Committee of the Affiliated Hospital of North Sichuan Medical College (approval no. NSMC201901).

\section{Patient consent for publication}

Not applicable.

\section{Competing interests}

The authors declare that they have no competing interests.

\section{References}

1. Wang Z, Zhou W, Dong H, Ma X and He Z: Dexmedetomidine pretreatment inhibits cerebral ischemia/reperfusioninduced neuroinflammation via activation of AMPK. Mol Med Rep 18: 3957-3964, 2018

2. GBD 2017 Causes of Death Collaborators: Global, regional, and national age-sex-specific mortality for 282 causes of death in 195 countries and territories, 1980-2017: A systematic analysis for the Global Burden of Disease Study 2017. Lancet 392: 1736-1788, 2018.

3. Jung JE, Kim GS, Chen H, Maier CM, Narasimhan P, Song YS, Niizuma K, Katsu M, Okami N, Yoshioka H, et al: Reperfusion and neurovascular dysfunction in stroke: From basic mechanisms to potential strategies for neuroprotection. Mol Neurobiol 41: 172-179, 2010.

4. Zhao $\mathrm{Y}$ and $\mathrm{Xu}$ J: Sanggenon $\mathrm{C}$ Ameliorates cerebral Ischemia-Reperfusion injury by inhibiting inflammation and oxidative stress through regulating RhoA-ROCK signaling. Inflammation 43: 1476-1487, 2020.

5. Esenwa CC and Elkind MS: Inflammatory risk factors, biomarkers and associated therapy in ischaemic stroke. Nat Rev Neurol 12: 594-604, 2016.

6. Yin F, Zhou H, Fang Y, Li C, He Y, Yu L, Wan H and Yang J: Astragaloside IV alleviates ischemia reperfusion-induced apoptosis by inhibiting the activation of key factors in death receptor pathway and mitochondrial pathway. J Ethnopharmacol 248: 112319,2020

7. Brosius AD, Ziller JW and Zhang Q: Relative and absolute configuration of aloperine. Acta Crystallogr C 53 (Pt 10): 1510-1512, 1997.

8. Zhao P, Zhou R, Zhu XY, Hao YJ, Li N, Wang J, Niu Y, Sun T, Li YX and Yu JQ: Matrine attenuates focal cerebral ischemic injury by improving antioxidant activity and inhibiting apoptosis in mice. Int J Mol Med 36: 633-644, 2015.

9. Zhao J,Zhang G,Li M,Luo Q,Leng Y and Liu X: Neuro-protective effects of aloperine in an Alzheimer's disease cellular model. Biomed Pharmacother 108: 137-143, 2018.

10. Hu S, Zhang Y, Zhang M, Guo Y, Yang P, Zhang S, Simsekyilmaz S, Xu JF, Li J, Xiang X, et al: Aloperine protects mice against Ischemia-Reperfusion (IR)-induced renal injury by regulating $\mathrm{PI} 3 \mathrm{~K} / \mathrm{AKT} / \mathrm{mTOR}$ signaling and AP-1 activity. Mol Med 21: 912-923, 2016.

11. Ma NT, Zhou R, Chang RY, Hao YJ, Ma L, Jin SJ, Du J, Zheng J, Zhao CJ, Niu Y, et al: Protective effects of aloperine on neonatal rat primary cultured hippocampal neurons injured by oxygen-glucose deprivation and reperfusion. J Nat Med 69: 575-583, 2015.

12. Song S, Chen Y, Han F, Dong M, Xiang X, Sui J, Li Y, Yang H and Liu J: Aloperine activates the Nrf2-ARE pathway when ameliorating early brain injury in a subarachnoid hemorrhage model. Exp Ther Med 15: 3847-3855, 2018.

13. Wang M, Chen Z, Yang L and Ding L: Sappanone A protects against inflammation, oxidative stress and apoptosis in cerebral Ischemia-Reperfusion injury by alleviating endoplasmic reticulum stress. Inflammation 44: 934-945, 2021.

14. Wu F, Yao W, Yang J, Zhang M, Xu Y, Hao Y, Yan L, Niu Y, Sun T, Yu J and Zhou R: Protective effects of aloperin on monocroline-induced pulmonary hypertension via regulation of Rho A/Rho kinsase pathway in rats. Biomed Pharmacother 95: 1161-1168, 2017.

15. Song G, Huang Y, Xiong M, Yang Z, Liu Q, Shen J, Zhao P and Yang X: Aloperine relieves Type 2 diabetes mellitus via enhancing GLUT4 expression and translocation. Front Pharmacol 11: 561956, 2021.

16. Bork K, Wurm F, Haller H, Strauss C, Scheller C, Gnanapragassam VS and Horstkorte R: Neuroprotective and neuroregenerative effects of nimodipine in a model system of neuronal differentiation and neurite outgrowth. Molecules 20: 1003-1013, 2015

17. Moran JM and Pedrera-Zamorano JD: Comments on 'Efficacy and safety assessment of acupuncture and nimodipine to treat mild cognitive impairment after cerebral infarction: A randomized controlled trial'. BMC Complement Altern Med 17: 119, 2017. 
18. Chen X, Yao Z, Peng X, Wu L, Wu H, Ou Y and Lai J: Eupafolin alleviates cerebral ischemia/reperfusion injury in rats via blocking the TLR4/NF- $\mathrm{BB}$ signaling pathway. Mol Med Rep 22: 5135-5144, 2020

19. Sanz JM, Chiozzi P, Colaianna M, Zotti M, Ferrari D, Trabace L, Zuliani G and Di Virgilio F: Nimodipine inhibits IL-1beta release stimulated by amyloid beta from microglia. Br J Pharmacol 167: $1702-1711,2012$

20. Han K, Rong W, Wang Q, Qu J, Li Q, Bi K and Liu R: Time-dependent metabolomics study of cerebral ischemia-reperfusion and its treatment: Focus on the combination of traditional Chinese medicine and Western medicine. Anal Bioanal Chem 412: 7195-7209, 2020.

21. Zou S, Zhang M, Feng L, Zhou Y, Li L and Ban L: Protective effects of notoginsenoside R1 on cerebral ischemia-reperfusion injury in rats. Exp Ther Med 14: 6012-6016, 2017.

22. Trotman-Lucas M, Kelly ME, Janus J and Gibson CL: Middle cerebral artery occlusion allowing reperfusion via common carotid artery repair in mice. J Vis Exp 2019.

23. Shi Y, Peng XH, Li X, Luo GP and Wu MF: Neuroprotective role of dexmedetomidine pretreatment in cerebral ischemia injury via ADRA2A-mediated phosphorylation of ERK1/2 in adult rats Exp Ther Med 16: 5201-5209, 2018.

24. Hu Q, Chen C, Yan J, Yang X, Shi X, Zhao J, Lei J, Yang L, Wang K, Chen L, et al: Therapeutic application of gene silencing MMP-9 in a middle cerebral artery occlusion-induced focal ischemia rat model. Exp Neurol 216: 35-46, 2009.

25. Hao MQ, Xie LJ, Leng W and Xue RW: Trim47 is a critical regulator of cerebral ischemia-reperfusion injury through regulating apoptosis and inflammation. Biochem Biophys Res Commun 515: 651-657, 2019.

26. Park SJ, Nam KW, Lee HJ, Cho EY, Koo U and Mar W: Neuroprotective effects of an alkaloid-free ethyl acetate extract from the root of Sophora flavescens Ait. against focal cerebral ischemia in rats. Phytomedicine 16: 1042-1051, 2009.

27. Zhao P, Zhou R, Li HN, Yao WX, Qiao HQ, Wang SJ, Niu Y, Sun T, Li YX and Yu JQ: Oxymatrine attenuated hypoxic-ischemic brain damage in neonatal rats via improving antioxidant enzyme activities and inhibiting cell death. Neurochem Int 89: $17-27,2015$.

28. Peters O, Back T, Lindauer U, Busch C, Megow D, Dreier J and Dirnagl U: Increased formation of reactive oxygen species after permanent and reversible middle cerebral artery occlusion in the rat. J Cereb Blood Flow Metab 18: 196-205, 1998.

29. Yang Z, Weian C, Susu H and Hanmin W: Protective effects of mangiferin on cerebral ischemia-reperfusion injury and its mechanisms. Eur J Pharmacol 771: 145-151, 2016.

30. Wang Q, Sun AY, Simonyi A, Jensen MD, Shelat PB Rottinghaus GE, MacDonald RS, Miller DK, Lubahn DE, Weisman GA and Sun GY: Neuroprotective mechanisms of curcumin against cerebral ischemia-induced neuronal apoptosis and behavioral deficits. J Neurosci Res 82: 138-148, 2005.

31. Wang TF, Lei Z, Li YX, Wang YS, Wang J, Wang SJ, Hao YJ, Zhou R, Jin SJ, Du J, et al: Oxysophoridine protects against focal cerebral ischemic injury by inhibiting oxidative stress and apoptosis in mice. Neurochem Res 38: 2408-2417, 2013.

32. Zhang B, Zhong Q, Chen X, Wu X, Sha R, Song G, Zhang C and Chen X: Neuroprotective effects of celastrol on transient global cerebral ischemia rats via regulating $\mathrm{HMGB} 1 / \mathrm{NF}-\mathrm{kB}$ signaling pathway. Front Neurosci 14: 847, 2020.

33. Ahmad A, Khan MM, Raza SS, Javed H, Ashafaq M, Islam F, Safhi MM and Islam F: Ocimum sanctum attenuates oxidative damage and neurological deficits following focal cerebral ischemia/reperfusion injury in rats. Neurol Sci 33: 1239-1247, 2012.

34. Wei W, Lan XB, Liu N, Yang JM, Du J, Ma L, Zhang WJ, Niu JG Sun T and Yu JQ: Echinacoside alleviates hypoxic-ischemic brain injury in neonatal rat by enhancing antioxidant capacity and inhibiting apoptosis. Neurochem Res 44: 1582-1592, 2019.
35. Wu F, Hao Y, Yang J, Yao W, Xu Y, Yan L, Niu Y, Sun T, Yu J and Zhou R: Protective effects of aloperine on monocrotaline-induced pulmonary hypertension in rats. Biomed Pharmacother 89: 632-641, 2017.

36. Zhang J, Zhou H, Chen J, Lv X and Liu H: Aloperine protects human retinal pigment epithelial cells against hydrogen peroxide-induced oxidative stress and apoptosis through activation of Nrf2/HO-1 pathway. J Recept Signal Transduct Res: Nov 30, 2020 (Epub ahead of print).

37. Han B, Lu Y, Zhao H, Wang Y, Li L and Wang $T$ Electroacupuncture modulated the inflammatory reaction in MCAO rats via inhibiting the TLR4/NF- $\mathrm{BB}$ signaling pathway in microglia. Int J Clin Exp Pathol 8: 11199-11205, 2015.

38. Hou SZ, Li Y, Zhu XL, Wang ZY, Wang X and Xu Y: Ameliorative effects of diammonium glycyrrhizinate on inflammation in focal cerebral ischemic-reperfusion injury. Brain Res 1447: 20-27, 2012.

39. Shichita T, Sakaguchi R, Suzuki M and Yoshimura A: Post-ischemic inflammation in the brain. Front Immunol 3: 132, 2012.

40. Liu Q and Zhang Y: PRDX1 enhances cerebral ischemia-reperfusion injury through activation of TLR4-regulated inflammation and apoptosis. Biochem Biophys Res Commun 519: 453-461, 2019.

41. Kao TK, Ou YC, Liao SL, Chen WY, Wang CC, Chen SY, Chiang AN and Chen CJ: Opioids modulate post-ischemic progression in a rat model of stroke. Neurochem Int 52 1256-1265, 2008

42. Zhao J, Li L and Fang G: Salvianolic acid A attenuates cerebral ischemia/reperfusion injury induced rat brain damage, inflammation and apoptosis by regulating miR-499a/DDK1. Am J Transl Res 12: 3288-3301, 2020.

43. Chang Z, Zhang P, Zhang M, Jun F, Hu Z, Yang J, Wu Y and Zhou R: Aloperine suppresses human pulmonary vascular smooth muscle cell proliferation via inhibiting inflammatory response. Chin J Physiol 62: 157-165, 2019.

44. Mao Q, Guo F, Liang X, Wu Y and Lu Y: Aloperine activates the PI3K/Akt Pathway and protects against coronary microembolisation-induced myocardial injury in rats. Pharmacology 104: 90-97, 2019.

45. Cantley LC: The phosphoinositide 3-kinase pathway. Science 296: 1655-1657, 2002.

46. Feng C, Wan H, Zhang Y, Yu L, Shao C, He Y, Wan H and Jin W: Neuroprotective effect of Danhong injection on cerebral Ischemia-Reperfusion injury in rats by activation of the PI3K-Akt pathway. Front Pharmacol 11: 298, 2020.

47. Yu Y, Jia XJ, Zong QF, Zhang GJ, Ye HW, Hu J, Gao Q and Guan SD: Remote ischemic postconditioning protects the heart by upregulating ALDH2 expression levels through the PI3K/Akt signaling pathway. Mol Med Rep 10: 536-542, 2014.

48. Wang Z, Han Y, Tian S, Bao J, Wang Y and Jiao J: Lupeol alleviates cerebral Ischemia-Reperfusion injury in correlation with modulation of PI3K/Akt pathway. Neuropsychiatr Dis Treat 16: 1381-1390, 2020.

49. Pignataro G, Meller R, Inoue K, Ordonez AN, Ashley MD, Xiong Z, Gala R and Simon RP: In vivo and in vitro characterization of a novel neuroprotective strategy for stroke: Ischemic postconditioning. J Cereb Blood Flow Metab 28: 232-241, 2008.

50. Fu X, Sun F, Wang F, Zhang J, Zheng B, Zhong J, Yue T, Zheng X, Xu JF and Wang CY: Aloperine protects mice against DSS-induced colitis by PP2A-mediated PI3K/Akt/mTOR signaling suppression. Mediators Inflamm 2017: 5706152, 2017.

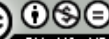

This work is licensed under a Creative Commons Attribution-NonCommercial-NoDerivatives 4.0 International (CC BY-NC-ND 4.0) License. 